
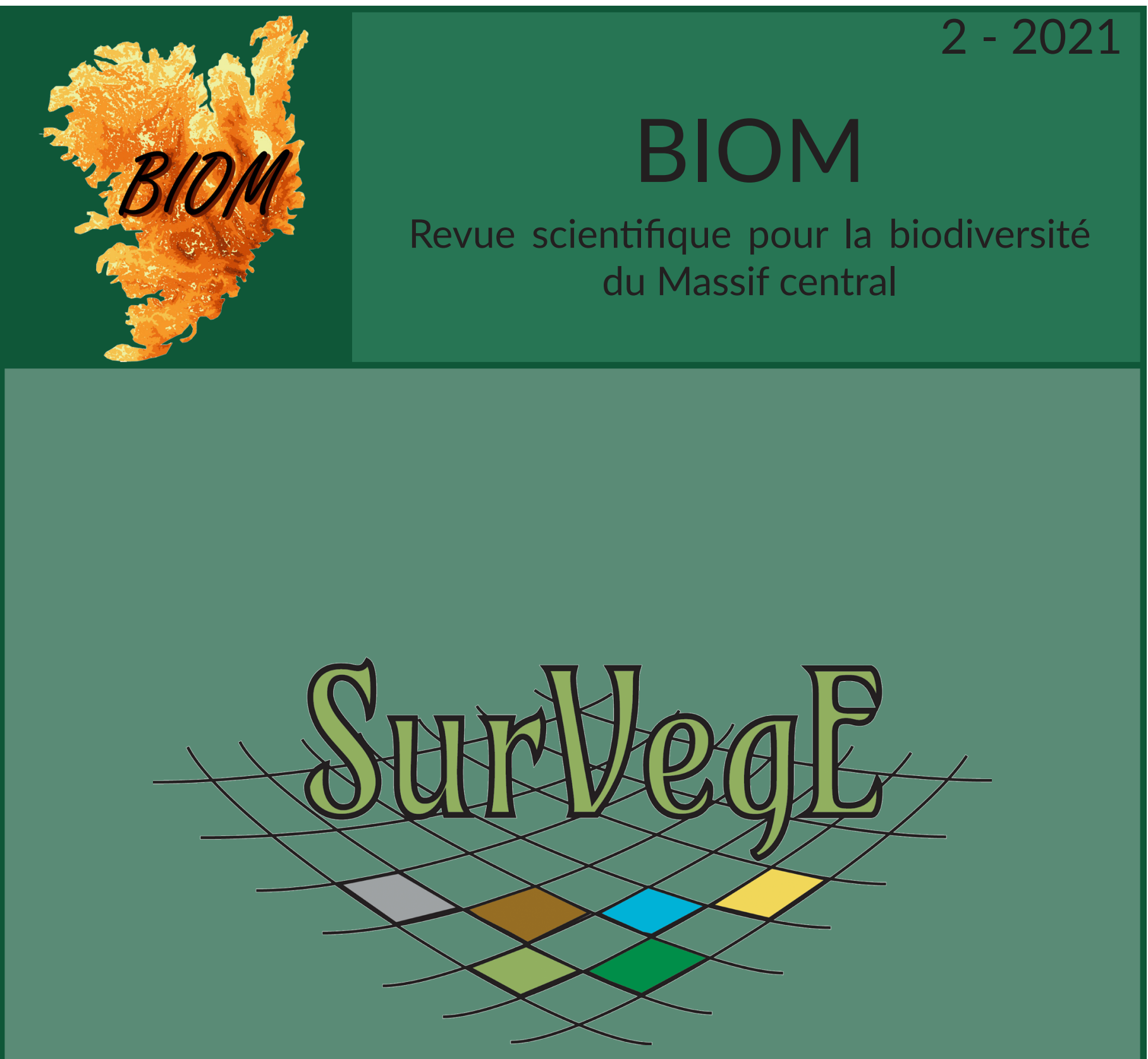

SurVegE-logiciel pour le suivi de la végétation en parcelles permanentes

Roux \& Thébaud / BIOM 2 (2021) : 51-61 


\title{
SurVegE-logiciel pour le suivi de la végétation en parcelles permanentes
}

\author{
Camille Roux ${ }^{1} \&$ Gilles Thébaud ${ }^{1}$
}

\author{
${ }^{1}$ Université Clermont Auvergne, UniVegE, F-63000 Clermont-Ferrand - camille.roux@uca.fr \\ ${ }^{1}$ Université Clermont Auvergne, UniVegE, F-63000 Clermont-Ferrand - gilles.thebaud@uca.fr
}

Soumis le 22 février 2021

Accepté le 3 avril 2021

Publié le 1 juin 2021
Mots-clés

Bio-indicateur

Suivi

Espaces naturels

Étude diachronique

Gestion

Keywords

Bio-indicator

Monitoring

Natural areas

Diachronic study

Management

\section{Résumé}

UniVegE, Université Clermont Auvergne, conduit des recherches qui ont trait à la caractérisation des habitats naturels et anthropisés, à partir des bio-indicateurs végétaux, dans une finalité de gestion et de conservation. Depuis 2005, ces travaux ont été complétés par des recherches et expertises portant sur le diagnostic et le suivi d'espaces naturels sur des parcelles permanentes et le long de transects, pour le compte de partenaires de l'environnement : Parcs naturels régionaux, Espaces Naturels Sensibles du département 63, Clermont-Auvergne métropole... Dans ce cadre 51 parcelles permanentes ont été mises en place dans des espaces naturels du Massif central, territoires de collectivités territoriales et/ou aires protégées. Cette expérience a permis de tester de nouvelles méthodes de suivi pour répondre concrètement aux questionnements des gestionnaires et déboucher sur l'élaboration du logiciel SurVegE.

SurVegE (SURveillance VÉGétation Écologie) est un logiciel d'assistance à l'opérateur gestionnaire d'espaces naturels. II permet, à partir de relevés floristiques effectués sur des parcelles permanentes bornées et géoréférencées, de caractériser les changements floristiques et écologiques intervenus entre plusieurs années. L'analyse des données, intégrée dans le logiciel et basée sur des valeurs d'indices de référence affectés aux espèces végétales, fournit directement les résultats comparatifs chiffrés et illustrés, sous forme de diagrammes, figures et tableaux.

Nous allons présenter ici certaines fonctionnalités du logiciel en prenant comme exemple des cas concrets d'études menées sur le terrain. Les sites sélectionnés correspondent à différents types d'habitats et ont fait l'objet soit d'états initiaux, soit d'analyses diachroniques comparatives.

\section{Abstract}

UniVegE, Clermont Auvergne University, conducts research relating to the characterization of natural and anthropized habitats, from plant bio-indicators, for the purpose of management and conservation. Since 2005 , this work has been supplemented by research and expertise relating to the diagnosis and monitoring of natural areas on permanent plots and along transects, on behalf of environmental partners: Regional nature parks, Espaces Naturels Sensibles of the departement 63, Clermont-Auvergne metropole ... In this context 51 permanent plots have been set up in natural areas of the Massif central, territories of local authorities and/or protected areas. This experience made it possible to test new monitoring methods to concretely respond to managers' questions and lead to the development of the SurVegE software.

SurVegE (SURvey VEGetation Ecology) is an assistance software for the operator who manages natural areas. It allows, from floristic surveys carried out on bounded and georeferenced permanent plots, to characterize the floristic and ecological changes occurring between several years. The data analysis, integrated into the software and based on the values of benchmarks assigned to plant species, directly provides the comparative results quantified and illustrated, in the form of diagrams, figures and tables.

We will present here some features of the software, taking as an example concrete cases of studies carried out in the field. The sites selected correspond to different types of habitats and have been the subject of either initial reports or comparative diachronic analyses. 


\section{Introduction}

Afin d'arrêter ou de limiter le processus d'érosion de la biodiversité, de nombreux acteurs se sont mobilisés pour mettre en place des procédures adaptées. En Europe cette dynamique a débouché sur un plan d'action pour la protection de la diversité biologique et paysagère, avec la mise en place de la directive habitats. Celle-ci a pour but de promouvoir la protection et la gestion des espaces naturels à valeur patrimoniale par l'intermédiaire du réseau Natura 2000. L'union européenne procède ainsi à une évaluation tous les six ans de l'application de la directive " habitats faune-flore " de 1992 (Directive $\mathrm{n}^{\circ}$ 92/43/CEE) (DHFF), sous forme de rapports demandés aux états membres, sur l'état de conservation des habitats et des espèces (Bensettiti et al. 2009, 2015). Pour chaque site local impliqué dans ces programmes européens de la DHFF, des documents d'objectifs, comprenant une évaluation de la biodiversité actuelle, des menaces associées et des lignes directrices pour la gestion locale, sont produits à intervalles réguliers pour améliorer la gestion des espaces naturels concernés.

En France, de nombreux programmes ou procédures concernant la préservation de la biodiversité, des habitats et des espaces naturels sont portés par différents acteurs : parcs nationaux, réserves naturelles nationales, réserves naturelles régionales (conseils régionaux), Espaces Naturels Sensibles (départements), acquisition foncière et gestion conservatoire (conservatoires d'espaces naturels, parcs naturels régionaux, conservatoires botaniques nationaux...). Ces espaces naturels requièrent également une surveillance temporelle assidue et afin de les gérer au mieux, des méthodes et outils de suivi et d'évaluation de l'évolution de la biodiversité sont mis en place (Daget \& Poissonet 2010).

Pour apprécier la dynamique de la biodiversité et les variations quantitatives et qualitatives des espèces végétales et de leurs habitats, il est nécessaire de mettre en place des méthodes de suivi diachroniques sur des parcelles permanentes (Chytrý et al. 2009). Plusieurs techniques sont utilisées pour effectuer les relevés sur ces parcelles : relevés floristiques, relevés phytosociologiques (Braun-Blanquet 1932, 1964), quadrats... Celles-ci peuvent être couplées avec l'utilisation de bio-indicateurs : indices écologiques (Ellenberg et al. 1992), indice de valeur fourragère (Daget \& Poissonnet 1972), groupes écologiques (Duvigneaud 1946) et beaucoup de travaux sont développés dans ce sens depuis de nombreuses années (Hawkes et al. 1997 ; Bartoli et al. 2000 ; Gégout et al. 2005 ; Hill \& Carey 2009 ; Gaudin 2012).

C'est dans un objectif d'intégration et d'automatisation de ces méthodes, largement partagées, qu'a été réalisé le logiciel SurVegE, afin d'apporter pour les suivis de végétation un diagnostic et une analyse comparative diachronique et multifactorielle au moyen des bio-indicateurs végétaux.

C'est un outil qui donne rapidement, à partir des relevés réalisés, des résultats phyto-écologiques variés permettant d'apporter assistance et conseil aux gestionnaires et conservateurs d'espaces et de milieux naturels ou anthropisés. Sa mise en œuvre nécessite des opérateurs de terrain compétents en botanique. II a été réalisé par UniVegE, de l'Université Clermont Auvergne et est le fruit de recherches conduites sur le terrain depuis 2005 (Thébaud et al. 2012 ; Roux 2017). SurVegE est un outil qui permet à la fois le diagnostic phyto-écologique et le suivi diachronique. II peut n'être utilisé que dans la première perspective. Mais son intérêt essentiel est de regrouper les deux.

Depuis 15 ans, 69 parcelles permanentes ont été mises en place dans 53 sites (Supplément 1) localisés dans plusieurs départements du Massif central (Puy-de-Dôme, Cantal, Haute-Loire, Loire et Nièvre). Plusieurs types d'habitats sont concernés : tourbières, marais, landes et pelouses submontagnardes et montagnardes, prairies, coteaux xériques de plaines, bords d'étangs/lacs, végétations alluviales et forêts. Ces parcelles se trouvent dans des espaces naturels, territoires de collectivités territoriales (parcs naturels, départements) et/ou aires protégées (réserves naturelles régionales/nationales, arrêtés de protection de biotope...). Ces zones ont fait l'objet d'un diagnostic phyto-écologique dans le cadre d'états initiaux : caractérisation des communautés végétales et des habitats, leur enveloppe écologique, recensement des espèces à statut patrimonial, usages et gestion. Et depuis 2012, des analyses diachroniques comparatives ont été effectuées sur certains sites, détaillées dans différents rapports d'études. Un article méthodologique présentant le logiciel est en cours de publication (Roux \& Thébaud, soumis).

Nous allons voir dans ce présent article différentes applications proposées par le logiciel au travers de cas concrets. Les données obtenues lors d'études concernant plusieurs sites et habitats, sont analysées et la démarche pour obtenir le diagnostic fourni aux gestionnaires des sites est expliquée.

\section{Méthodologie}

\section{Structure générale du logiciel}

La base de données actuellement intégrée au logiciel est formée à partir du référentiel taxonomique TaxRef v.12 (Gargominy et al. 2018) complété par les valeurs des indices d'Ellenberg (1992) et, pour les taxons non renseignés, les indices écologiques de Julve (2017) sont adoptés, sauf exceptions qui sont alors documentées par UniVegE. Certaines bryophytes, notamment turficoles, sont documentées par des valeurs d'indices calculés par UniVegE à partir de Dierssen (2001). Pour les taxons des milieux agropastoraux les indices spécifiques des valeurs fourragères proviennent de Daget \& Poisonnet (2010). Tous les taxons n'ont pas forcément des valeurs d'indices renseignés mais le logiciel permet de compléter celles-ci.

SurVegE fonctionne sur Windows (à partir de Windows 7) et Linux, en local. Le logiciel exporte les illustrations aux formats bitmap et vectorisé afin de pouvoir les retravailler, si besoin, sur des logiciels spécialisés. Et les tableaux sont exportés au format csv.

Les relevés floristiques sont saisis directement dans le logiciel, mais il est possible de les importer de Turboveg (SDF formated database) (Hennekens \& Schaminée 2001).

Les explications détaillées du logiciel sont fournies dans un guide d'utilisation. 

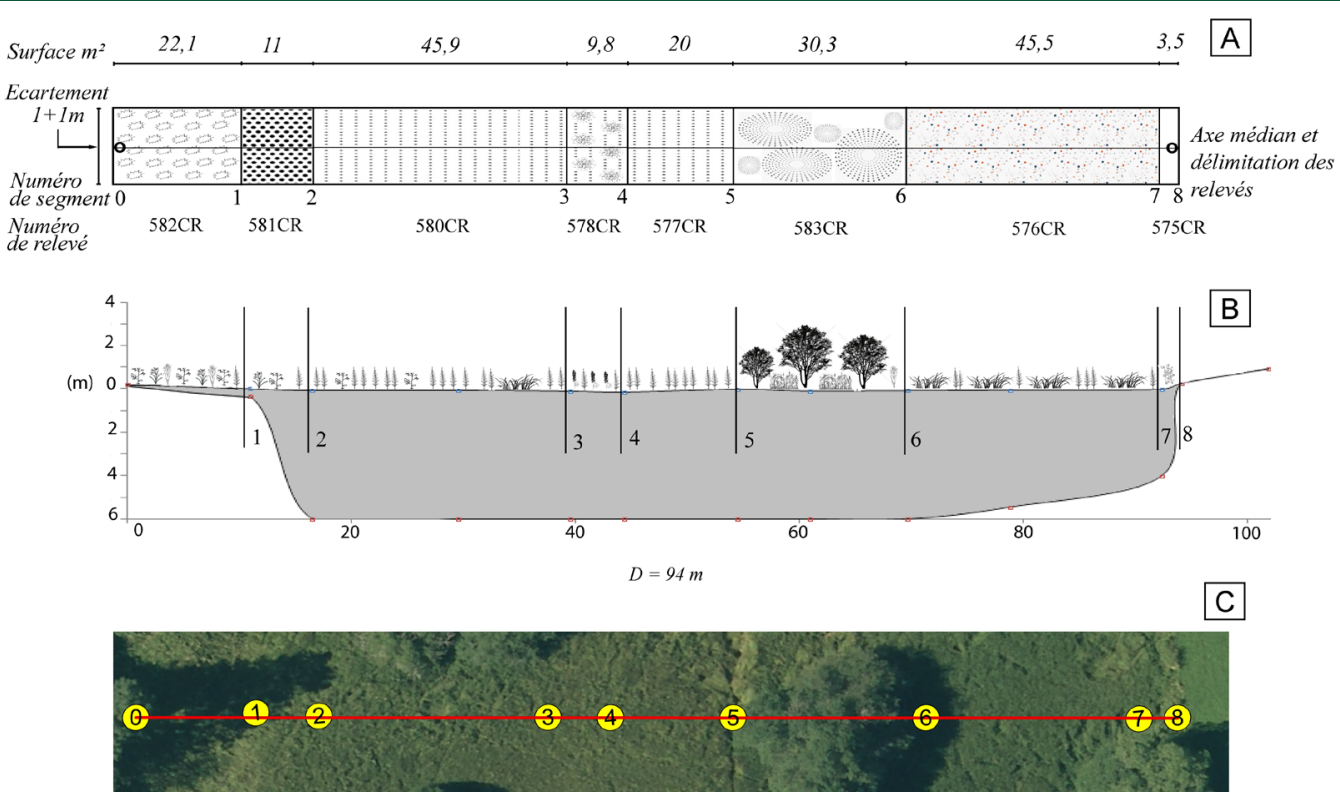

Figure 1 - A) : toposéquence de végétation étudiée vue de dessus ; avec, de haut en bas : les zones enregistrées, les points délimitant les différentes sections ; les numéros de relevé correspondants. B) : toposéquence de la même végétation en profil en coupe ; le profil topographique, la profondeur de la tourbe (cette dernière uniquement dans ce cas particulier) avec des échelles de hauteur et de distance. C) : visualisation de la séquence sur la photo aérienne.

\section{Mise en place de parcelles permanentes}

La méthode consiste en la mise en place sur le terrain d'un PSET (profil socio-écologique temporel ; Fig. 1), séquence permanente composée de plusieurs segments, correspondant à une surface donnée, bornés et géoréférencés, dans lesquels sont effectués des relevés de végétation au cours du temps. Dans le cas de la réalisation de levées topographiques le long du PSET on utilise un appareil de type tachéomètre. On mesure ainsi à la mire la hauteur de chaque point séparant les segments de végétation et leur distance horizontale par rapport au point zéro où sera placé le tachéomètre. Des mesures complémentaires peuvent être effectuées (profondeur de la tourbe par exemple...) que SurVegE permet d'illustrer.

Dans le cas où l'on ne réalise pas de profil topographique, il faudra néanmoins mesurer la distance au sol, par rapport au point zéro, entre les différents points de séparation des segments de végétation.

La réalisation d'un PSET est la méthode recommandée pour une utilisation optimale du logiciel SurVegE. Mais le logiciel est aussi adapté pour le suivi de simples relevés de végétation sur des parcelles isolées ou d'espèces végétales particulières.

Que les parcelles soient sous forme de transects linéaires ou de relevés non contigus, elles devront être géoréférencées par GPS et bornés par une borne métallique (ou aimant) enfoncée dans le sol. II est impératif de pouvoir, plusieurs années plus tard, retrouver très exactement ces points pour effectuer de nouveaux relevés dans les mêmes segments.

\section{Relevés de végétation}

Le logiciel est compatible avec plusieurs types de relevés de végétation, réalisés le long du PSET ou sur une parcelle permanente isolée : relevés phytosociologiques (Braun-Blanquet) ; relevés sur des points quadrats ; relevés phyto-écologiques de bioindicateurs ; relevés d'espèces individuellement.

Pour chaque relevé, en plus de l'identification taxonomique de toutes les espèces présentes, on relèvera les \% de couverture totale de la végétation, de chaque strate de végétation, de la litière, du sol nu et des blocs rocheux affleurants ; ainsi que les données stationnelles (altitude, exposition, pente...).

Pour renseigner l'abondance de chaque espèce, SurVegE propose plusieurs options : on peut noter le \% exact d'abondance, ou affecter un coefficient d'abondance selon Braun-Blanquet qui correspond à une classe d'abondance dont la valeur est estimée par rapport à la surface totale du relevé, variant de rà 5 . Ici encore le logiciel propose plusieurs options concernant les classes d'abondance : «Braun-Blanquet old ", "Braun-Blanquet new » et "Braun-Blanquet extend "; chacune déterminant un degré de précision de plus en plus fin des classes d'abondance (Tab. 1). Lors de la réalisation du relevé, la dominance peut-être notée, mais celle-ci ne sera pas renseignée dans le logiciel car elle n'intervient pas dans les calculs.

Tableau 1 - Choix des classes d'abondance proposées dans SurVegE.

\begin{tabular}{|c|c|c|c|c|c|c|c|c|}
\hline $\begin{array}{c}\text { Braun- } \\
\text { Blanquet old } \\
\text { Classe } \\
\end{array}$ & $\%$ & Moy & $\begin{array}{c}\text { Braun- } \\
\text { Blanquet new } \\
\text { Classe } \\
\end{array}$ & $\%$ & Moy & $\begin{array}{c}\text { Braun- } \\
\text { Blanquet } \\
\text { extend Classe }\end{array}$ & $\%$ & Moy \\
\hline 5 & ]75-100] & 87.5 & 5 & ]75-100] & 87.5 & $5 b$ & ]87.5-100] & 93.75 \\
\hline 4 & ]50-75] & 62.5 & 4 & ]50-75] & 62.5 & $5 a$ & ]75-87.5] & 81.25 \\
\hline 3 & ]25-50] & 37.5 & 3 & ]25-50] & 37.5 & $4 b$ & ]62.5-75] & 68.75 \\
\hline 2 & ]5-25] & 15 & $2 b$ & ]15-25] & 20 & $4 a$ & ]50-62.5] & 56.25 \\
\hline 1 & ]1-5] & 3 & $2 a$ & ]5-15] & 10 & $3 b$ & ]37.5-50] & 43.75 \\
\hline+ & 0.5 & 0.5 & 1 & ]1-5] & 3 & $3 a$ & ]25-37.5] & 31.25 \\
\hline \multirow[t]{5}{*}{$r$} & 0.1 & 0.1 & + & 0.5 & 0.5 & $2 b$ & ]15-25] & 20 \\
\hline & & & $r$ & 0.1 & 0.1 & $2 a$ & ]5-15] & 10 \\
\hline & & & & & & 1 & ]1-5] & 3 \\
\hline & & & & & & + & 0.5 & 0.5 \\
\hline & & & & & & $r$ & 0.1 & 0.1 \\
\hline
\end{tabular}


Les relevés effectués selon la méthode de Braun-Blanquet, c'est-à-dire en classes d'abondance, sont moins précis que les relevés d'abondance en valeur absolue. Mais les premiers ne nécessitent qu'un tiers à un cinquième du temps de terrain requis pour les seconds et fournissent des données de base suffisamment précises pour permettre l'évaluation de l'impact sur l'environnement (Wikum \& Shanholtzer 1978). Ils permettent également une identification directe de la communauté végétale concernée selon le synsystème européen (Mucina et al. 2016) ou selon le prodrome des végétations de France (PVF1/Bardat et al. 2004 et PVF2/Société Française de Phytosociologie). Ainsi qu'une caractérisation de l'habitat et de son affiliation à des directives communautaires ou des conventions internationales en utilisant par exemple HABREF qui regroupe les typologies de référence de chaque territoire (Clair et al. 2019).

\section{Utilisation de bio-indicateurs végétaux}

Les bio-indicateurs végétaux permettent, à partir des données floristiques collectées sur le terrain, de fournir des indications d'ordre écologique permettant le diagnostic de l'état initial et de l'évolution du site étudié. Chaque taxon possède une valeur d'indice et pour un relevé, le logiciel calcule la moyenne des valeurs des indices (lumière, humidité, température, $\mathrm{pH}$, nutriments, salinité et continentalité) pondérées par l'abondance des taxons. C'est pourquoi il est très important d'être particulièrement rigoureux lorsque l'on réalise un relevé par classes d'abondance, sous peine d'obtenir des résultats biaisés, voire incohérents.

\section{Saisie des données dans SurVegE}

Pour que le logiciel fonctionne il suffit de saisir les relevés de végétation effectués sur le terrain. II faut tout d'abord renseigner l'année de l'étude et le premier segment de la séquence, ensuite choisir la méthode retenue pour calculer l'abondance des taxons. Puis remplir les données de têtes du relevé de végétation : $n^{\circ}$ de relevé, données stationnelles, recouvrement, écartement, surface, géologie... On peut alors saisir le relevé, en précisant pour chaque taxon sa strate et son abondance. Pour chaque taxon saisi, on peut visionner les indices écologiques et les indices spécifiques de valeur fourragère qui lui correspondent et les compléter si nécessaire.

Dans le cas de la réalisation d'un PSET, on peut rentrer également la topographie du transect effectué sur le terrain. On renseigne l'altitude du premier point de la séquence, la localité, la commune, l'orientation générale de la séquence (liste déroulante) et le système de coordonnées utilisé (liste déroulante). II faut ensuite noter le numéro de point (en débutant par le point 0 , début du transect et en terminant par le dernier point) ; la hauteur relative en mètre renseignée pour chaque point est celle du point zéro (position du tachéomètre) soustraite (pente descendante) ou augmentée (pente montante) de la différence topographique observée grâce à la mire ; la distance cumulée à partir du point 0 est notée en mètre jusqu'au dernier point, on obtient ainsi la longueur totale de la séquence; ces données sont complétées par les coordonnées $X$ et $Y$ de chaque segment/relevé et éventuellement par la profondeur mesurée (sol, tourbe, eau) en mètre (Fig. 2).
II n'est pas nécessaire d'effectuer de mesures de topographie pour les différentes applications du logiciel, mais si l'on choisit la mise en place d'un transect, c'est-à-dire d'une séquence continue de plusieurs relevés, il faut néanmoins mesurer la distance au sol, par rapport au point zéro, entre les différents points de séparation des segments de végétation, afin d'obtenir la surface du relevé.

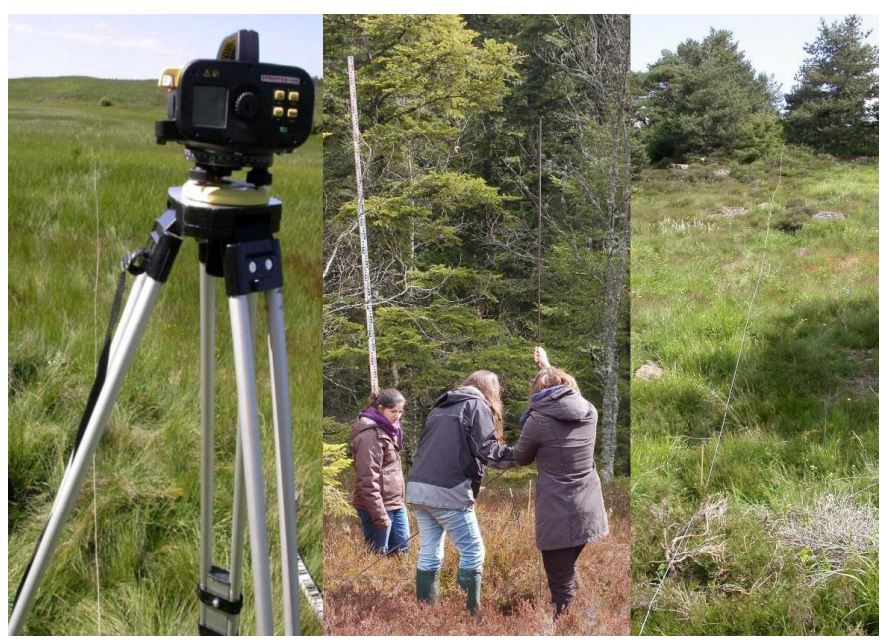

Figure 2 - Mise en place d'un PSET sur le terrain. De gauche à droite : niveau laser pour mesurer la distance entre chaque segment ; mire à code barre pour mesurer la hauteur et perche métallique graduée pour mesurer la profondeur de tourbe ; corde matérialisant la toposéquence au sol.

\section{Description des applications du logiciel à partir des résultats obtenus sur des sites de suivi}

SurVegE est utilisable pour tous types de milieux ; aussi avonsnous choisi comme exemples, pour présenter les fonctionnalités, des sites variés abritant divers habitats :

- Une tourbière acide à sphaignes, la tourbière de la Prénarde (Saint-Jean-Soleymieux, 42 ; ZNIEFF I n 820032401), fait l'objet d'un suivi sous la forme d'une toposéquence de $172 \mathrm{~m}$. qui a été mis en place en 2008 par UniVegE, afin d'analyser la libre évolution de la végétation après abandon du pâturage ; depuis la même période elle est étudiée sur les plans hydro-écologique et paléo-environnemental par le laboratoire ISTHME de l'université de Saint-Etienne.

- Un marais submontagnard, le marais de Fontfreyde (Saint-Genès-Champanelle, 63 ; site UNESCO FR7100006), situé dans le Parc Naturel Régional des Volcans d'Auvergne (PNRVA), a été identifié comme zone humide prioritaire sur le territoire de Clermont Auvergne Métropole, et celle-ci a confié une étude phyto-écologique et paléo-environnementale depuis 2012, aux universités de Clermont-Ferrand (UniVegE) et de Saint-Etienne (ISTHME) ; le suivi permanent correspond à une toposéquence de $102 \mathrm{~m}$.

- Une lande pastorale de lisière subalpine, sur la montagne de Monthiallier (Job, 63 ; ENS, NATURA 2000 FR8301030 ; ZNIEFF I n॰830005443 ; site classé du " Haut-Forez central "), située dans le Parc Naturel Régional du Livradois-Forez. Le Conseil départemental du Puy-de-Dôme a labellisé le site en Espace Naturel Sensible et a confié en 2012 à UniVegE un suivi afin d'y observer la dynamique de la végétation. Celui-ci correspond à une toposéquence de $45 \mathrm{~m}$.

- Une pelouse pastorale submontagnarde sur le puy de la 
Combegrasse (Aydat, 63 ; site UNESCO FR7100006 ; site classé de la " chaîne des Puys "), située dans le Parc Naturel Régional des Volcans d'Auvergne, dans la chaîne des Puys. Une étude a été commanditée à UniVegE par le PNRVA, en 2015 afin de suivre l'évolution de ces estives suite à un défrichage conduit par le Syndicat Mixte de Gestion Forestière d'Aydat. Ce suivi est réalisé sous la forme d'une toposéquence de $95 \mathrm{~m}$.

- Un coteau xérothermique calcaire de Limagne de haut intérêt botanique, puy Long (Clermont-Ferrand, 63 ; ZNIEFF I n830005667) situé sur le territoire de Clermont Auvergne Métropole. Celle-ci, dans le cadre du Plan biodiversité, a confié à UniVegE un suivi de la végétation depuis 2012 ; il correspond à une toposéquence de $56 \mathrm{~m}$.

- Un secteur de grèves de la rivière Allier, au lieu-dit « Les Boires " (Pont-du-Château, 63 ; NATURA 2000 FR8301038, ZNIEFF I n 830000178). La métropole a confié à UniVegE un suivi depuis 2013 afin d'observer la libre évolution du lit mineur. Celui-ci correspond à une toposéquence de $190 \mathrm{~m}$.

Ces sites présentent un gradient de pression anthropique variable. Certains sont en libre évolution depuis plus de 50 ans (Prénarde, Fontfreyde, les Boires) ; d'autres sont soumis à un pâturage extensif (Combegrasse, Monthiallier) ; le dernier site, en raison de sa proximité avec la métropole de Clermont-Ferrand, subit des impacts plus importants : fréquentation régulière de promeneurs, d'engins motorisés, proximité immédiate d'un ancien centre d'enfouissement technique, d'une déchetterie et d'une casse.

\section{Applications liées aux analyses phyto-écologiques}

\section{Production automatique d'un diagramme de répartition floristique}

SurVegE atteint son utilisation optimale dans le cas de la mise en place d'un profil de type PSET ; le logiciel fournit alors automatiquement un diagramme complet du transect de végétation (Fig. 3), avec en lignes, les taxons et en colonnes les différents relevés correspondant aux segments de la toposéquence, en respectant les proportions de distances. II permet ainsi de donner un diagnostic de l'état initial et de mettre en évidence indirectement, par le biais des taxons, des gradients écologiques, des successions dynamiques ou de rendre compte des contacts et transitions entre communautés. SurVegE permet aussi de modifier de manière automatique l'ordre des taxons qui apparaissent dans le diagramme selon leur valeur d'indice écologique ou en les classant manuellement, en déplaçant

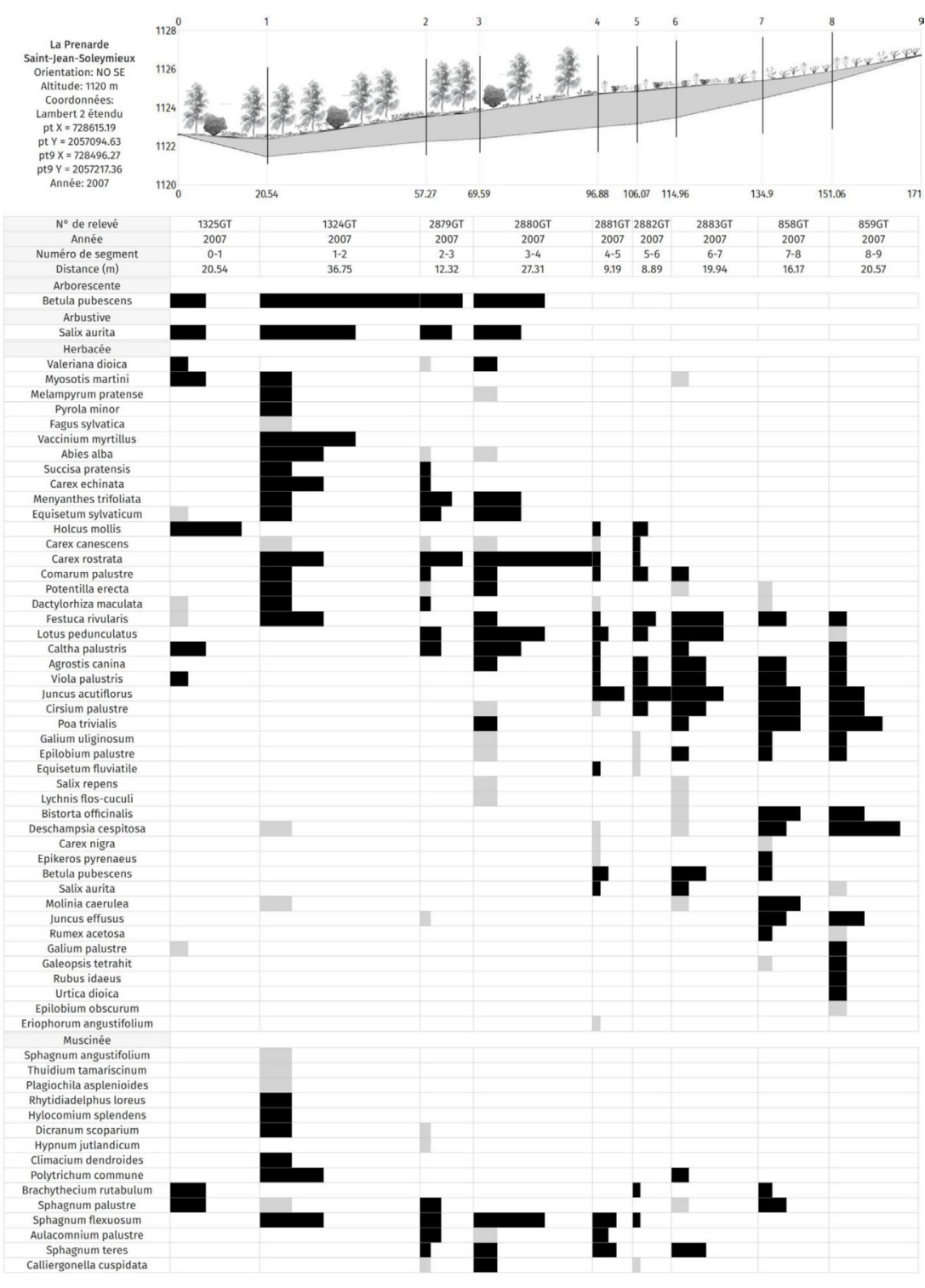

Figure 3 - Exemple d'un diagramme d'état initial de PSET effectué lors de l'année 2007 dans la tourbière de la Prénarde (Saint-JeanSoleymieux, 42). Profil topographique et profil de profondeur de tourbe. De 0 à 9 : différents segments du profil où sont effectués les relevés de végétation, situés chacun entre 2 points topographiques. Les espèces sont classées de manière à obtenir un gradient écologique, ici un gradient d'humidité décroissant de 2 à 9. Les illustrations du profil ne sont pas incorporées dans le logiciel mais rajoutées après exportation en .jpg ou .svg. 
les lignes correspondantes, de façon à produire un tableau diagonalisé des taxons.

Le diagramme de la figure 3 , réalisé dans la tourbière de la Prénarde, exprime après classement des taxons un gradient d'humidité décroissant de 2 à 9. II permet aussi de mettre en évidence deux compartiments fonctionnels et une succession dynamique. Un premier compartiment en amont (de 6 à 9), sur sols organo-minéral, sous l'influence du versant, mésotrophe et asséché en été, est formé de jonçaies à Juncus acutiflorus Ehrh. ex Hoffm. et prairies à Deschampsia cespitosa (L.) P.Beauv. (habitat d'intérêt communautaire UE 6410), mésohygrophiles, et un deuxième compartiment (de 1 à 6), plus régulièrement inondé, turfigène, riche en sphaignes et taxons hydrophiles, est occupé par des communautés de type bas-marais. Ce dernier est le siège d'une succession dynamique secondaire qui conduit, depuis les stades pionniers (3-4) à un stade plus mûr de la Boulaie (1-2). II conduira à une tête de série forestière turficole de type sapinière à bouleaux pubescents (Betulo pubescentis-Abietetum albae Lemée 1995, habitat d'intérêt communautaire UE 91D0), qui n'est pas encore pleinement réalisée sur ce profil ; déjà un certain assèchement en 1-2 est observable, qui s'accentuera lors de la croissance des sapins.

\section{Production automatique de résultats comparatifs quantitatifs à partir des valeurs d'indices}

L'emploi des indices écologiques dans le cadre de SurVegE permet d'obtenir deux types principaux d'informations :

- Comparaison phyto-écologique, pour une même année, entre chaque segment d'une toposéquence ou entre plusieurs relevés non contigus ;

- Comparaison diachronique à partir des changements de végétation observés.

SurVegE permet ainsi d'obtenir directement des résultats comparatifs pour chacun des indices sous forme de différentes illustrations et tableaux de valeurs associés. Nous développons brièvement ci-dessous trois exemples pour illustrer quelques types de résultats produits par le logiciel.

Exemple d'analyse diachronique sur le site de puy Long. Ce coteau pépéritique de Limagne est principalement occupé par des communautés de pelouses xérothermiques oligotrophiques, plus ou moins ouvertes (habitats d'intérêt communautaire UE 6110 et 6210$)$. L'analyse diachronique (2012-2019) a permis de constater une augmentation des occurrences et abondances de plantes nitrophiles et donc de l'indice des nutriments $(\mathrm{N})$ dans la majeure partie du transect (Fig. 4).

Seuls les segments 4-5 et 5-6 situés sur la pente sud, ont des valeurs d'indice moins importantes qu'en 2012, conséquence d'une érosion régulière du sol à cet endroit de forte pente. Cette augmentation de la teneur en nutriments le long du profil est peut-être une conséquence de retombées probables de composés organiques azotés, provenant de l'ancien centre d'enfouissement technique adjacent au site.

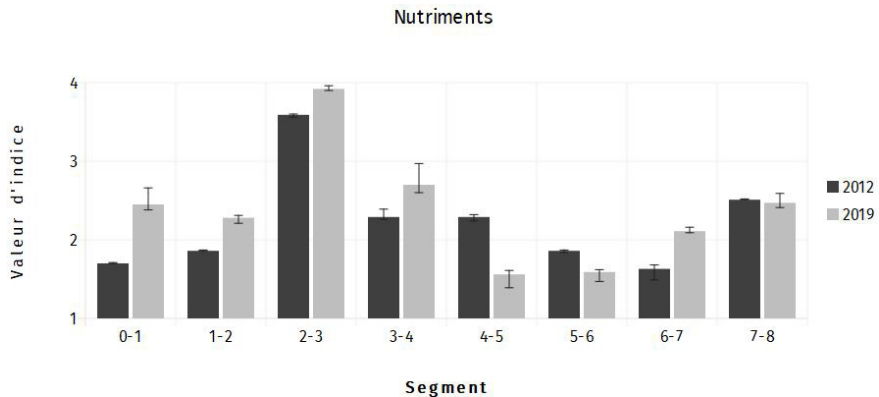

Figure 4 - Figure directement produite par le logiciel, correspondant à des histogrammes avec barres d'erreurs. Ils présentent la variation des moyennes des valeurs de l'indice des nutriments, entre 2012 et 2019, pour chacun des segments d'une toposéquence effectuée sur le site de puy Long (ClermontFerrand, 63). La barre d'erreur est obtenue en calculant l'IE du relevé avec le $\%$ d'abondance maximale et minimale de la classe d'abondance.

Exemple d'analyse diachronique du marais de Fontfreyde. La figure 5 montre la variation des valeurs de l'indice de température (T), entre 2012 et 2019, dans ce marais. On remarque dans cet exemple que cette augmentation est générale dans tous les segments. Le segment 3-4, au centre du marais, est le plus impacté, et c'est au niveau de la saulaie (5-6), milieu ombragé et tamponné, que le changement est le moins important. En étudiant plus précisément la composition floristique des segments (cf. paragraphe : Variations par taxon) nous avons constaté une disparition des espèces mésocryophiles montagnardes et une augmentation des espèces mésothermophiles submontagnardes à collinéennes, éléments qui viennent conforter et expliquer l'augmentation de l'indice T observée.

Température

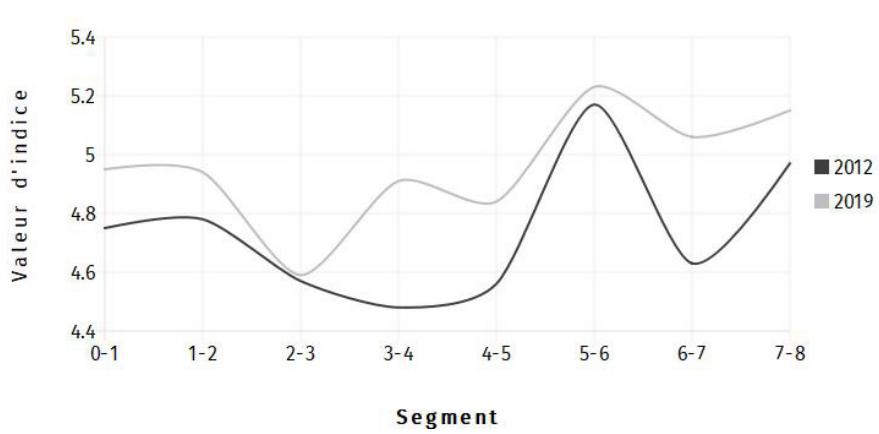

Figure 5 - Figure directement produite par le logiciel correspondant à des courbes présentant la variation des moyennes des valeurs de l'indice de température, entre 2012 et 2019, pour chacun des segments d'une toposéquence effectuée sur le marais de Fontfreyde. Ici l'axe des ordonnées est dynamique, mais il peut être exporté en échelle globale, c'est-à-dire qu'il affiche tous les indices présents pour une variable écologique, l'écart visuel entre les courbes devient donc moins prononcé.

Exemple de comparaison phyto-écologique de landes du Massif central. Dans cet exemple le logiciel permet de comparer plusieurs variables sur une même illustration et de donner directement, sous forme de figures en radar, le spectre écologique de quatre relevés effectués dans des situations stationnelles variées, éloignés les uns des autres, afin de mettre en évidence leurs différences. La figure 6 donne les profils effectués dans ces landes basses acidiphiles du Massif central (habitats d'intérêt communautaire 
UE 4030 et 4060). Les 3 premiers ont été réalisés dans l'étage subalpin, à des expositions différentes (deux dans les monts Dore et un dans les monts du Cantal) et le quatrième dans le Limousin à l'étage submontagnard.

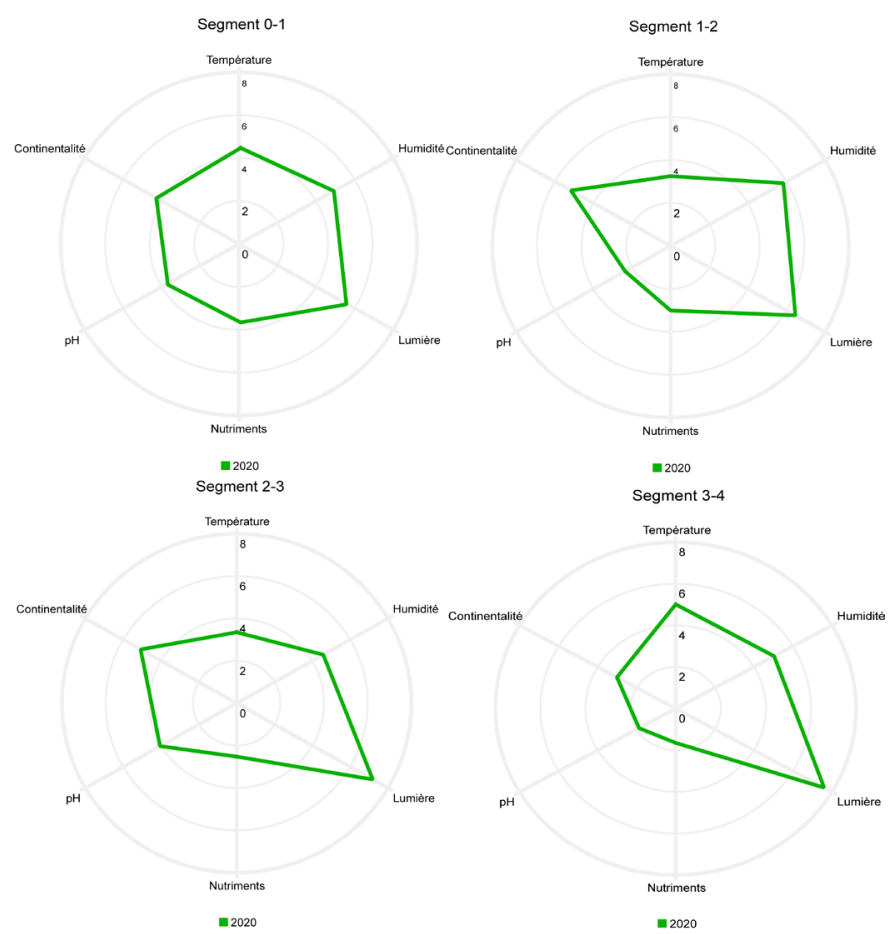

Figure 6 - Radars représentant les valeurs des indices écologiques (température, humidité, lumière, teneur en nutriments, réaction du sol et continentalité) de 4 landes situées dans le Massif central : 0-1 : lande chionophile subalpine des monts Dore ; 1-2 : lande cryosciaphile subalpine d'ubac des monts Dore ; 2-3 : lande hélioxérophile subalpine sommitale des monts du Cantal ; 4-5 : lande de basse altitude, humide et atlantique du Limousin.

En complément à ces résultats et illustrations, le logiciel SurVegE fournit le tableau des valeurs brutes des indices écologiques de la lumière, humidité, température, $\mathrm{pH}$, nutriments, salinité et continentalité, ainsi que les valeurs des indices de richesse spécifique, de Shannon et de régularité sous forme de tableau exportable depuis le logiciel (Tab. 2).

\section{Production de résultats sous forme de classes d'indice}

Cette fonctionnalité de SurVegE permet de regrouper les taxons de chaque segment selon leur classe d'indice (affinité écologique), pour chaque paramètre écologique. II apporte une aide à la compréhension et à l'interprétation des variations des valeurs des indices d'Ellenberg observées parallèlement dans un site donné.

On donne ici l'exemple du suivi de la toposéquence du marais de Fontfreyde. Le logiciel analyse la composition floristique de chaque segment, en classant les espèces selon leur affinité écologique pour le paramètre " humidité ». II fournit directement la répartition de chacune des classes d'indice, des plus sèches aux plus humides, le long de la toposéquence. La figure 7 montre les résultats, sous forme de courbes, pour les espèces de la classe d'indice 8 (mésohygrophiles à hygrophiles) et pour la classe d'indice 10 (hélophytes).

\section{Mésohyg rophile à hygrophile}
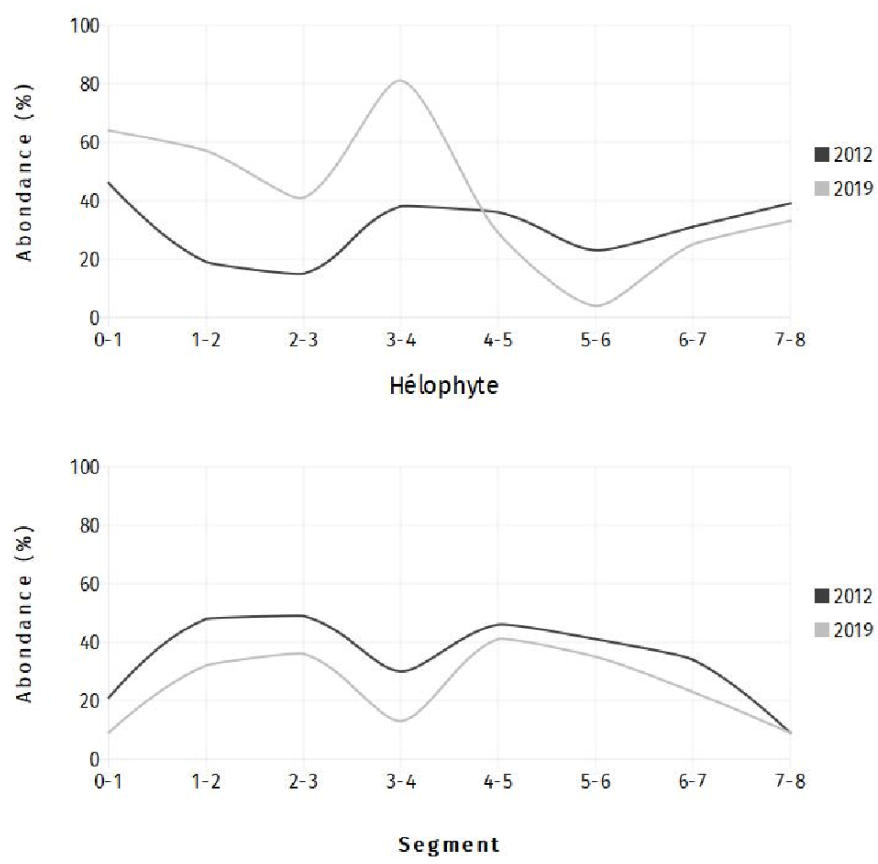

Figure 7 - Somme des abondances, en pourcentage, des taxons réunis par classe d'indice d'Ellenberg pour la variable « humidité » et par segment. Ici la comparaison des pourcentages d'abondance des taxons mésohygrophiles à hygrophiles (classe 8) et des hélophytes (classe 10) entre 2012 et 2019 au marais de Fontfreyde.

Tableau 2 - Exemple de tableau, directement produit par SurVegE, donnant les résultats des calculs sous forme des valeurs des indices écologiques ainsi que celles de l'indice de Shannon, la richesse spécifique et régularité, pour chaque segment/relevé.

\begin{tabular}{|c|c|c|c|c|c|c|c|c|c|c|c|}
\hline $\mathrm{N}^{\circ}$ de relevé & Année & Segment & Shannon-Wiener & Richesse & Régularité & Lumière & Température & Continentalité & Humidité & $\mathrm{pH}$ & Nutriments \\
\hline $582 \mathrm{CR}$ & 2019 & $0-1$ & 1.64 & 17 & 0.58 & 7.04 & 4.95 & 4.94 & 7.97 & 5.21 & 4.92 \\
\hline $581 C R$ & 2019 & $1-2$ & 1.7 & 12 & 0.69 & 7.2 & 4.94 & 4.67 & 8.68 & 5.43 & 5.34 \\
\hline $580 \mathrm{CR}$ & 2019 & $2-3$ & 1.87 & 20 & 0.62 & 7.6 & 4.59 & 4.5 & 8.83 & 4.66 & 4.14 \\
\hline $578 C R$ & 2019 & $3-4$ & 1.33 & 11 & 0.55 & 7.65 & 4.91 & 3.88 & 8.32 & 4.13 & 3.47 \\
\hline $577 C R$ & 2019 & $4-5$ & 1.88 & 16 & 0.68 & 7.63 & 4.84 & 4.57 & 9.05 & 5.4 & 4.81 \\
\hline $576 C R$ & 2019 & $5-6$ & 0.94 & 13 & 0.37 & 7.36 & 5.23 & 3.77 & 7.52 & 4.97 & 4.91 \\
\hline $575 \mathrm{CR}$ & 2019 & 6-7 & 1.69 & 14 & 0.64 & 7.07 & 5.06 & 4.73 & 8.96 & 6.28 & 5.85 \\
\hline $579 \mathrm{CR}$ & 2019 & $7-8$ & 2.1 & 16 & 0.76 & 6.43 & 5.15 & 3.81 & 7.65 & 5.55 & 5.78 \\
\hline
\end{tabular}


En 7 ans on constate dans le marais de Fontfreyde une baisse générale des valeurs de l'indice " humidité ». La figure 7 montre en 2019 une augmentation du pourcentage des espèces de classe 8 (mésohygrophiles à hygrophiles, irrégulièrement inondées) dans les 5 premiers segments de la séquence et parallèlement une diminution de celles de classe 10 (hélophytes, inondées en permanence). Ces résultats ont permis d'en déduire que la baisse générale de l'humidité observée était liée à une réduction de la période d'inondation du marais. Ceci entraîne un changement dans la composition de la végétation avec la mise en place d'une mégaphorbiaie à Filipendula ulmaria (L.) Maxim., avec Persicaria lapathifolia (L.) Delarbre (habitat UE 6430) au détriment de tremblants à Carex nigra (L.) Reichard et Equisetum fluviatile (habitat UE 7140), taxons dont les variations individuelles sont suivies par ailleurs (§ Variations par taxon).

\section{Production de résultats par strates et couvertures de végétation}

Cette application permet d'afficher l'abondance en pourcentage des couvertures végétales pour chacune des strates: arborescente, arbustive, herbacée et muscinale, pour le sol nu, la litière et les affleurements minéraux (rochers ...). En comparant plusieurs années on peut ainsi observer les changements structuraux de la végétation et de la surface recouverte.

On donne ici l'exemple d'une toposéquence effectuée en milieu alluvial, au bord de l'Allier au lieu-dit « Les Boires » (Pontdu-Château, 63). Elle se compose d'un ancien étang comblé récemment, d'ourlets forestiers, d'une forêt alluviale et de grèves exondées. Le suivi a été effectué sur un pas de temps de 6 ans (2013-2019) ; la figure 8 montre les changements structuraux observés, sous forme d'histogrammes affichant les valeurs de couvertures en \% pour chaque segment de la toposéquence en 2013 et 2019.

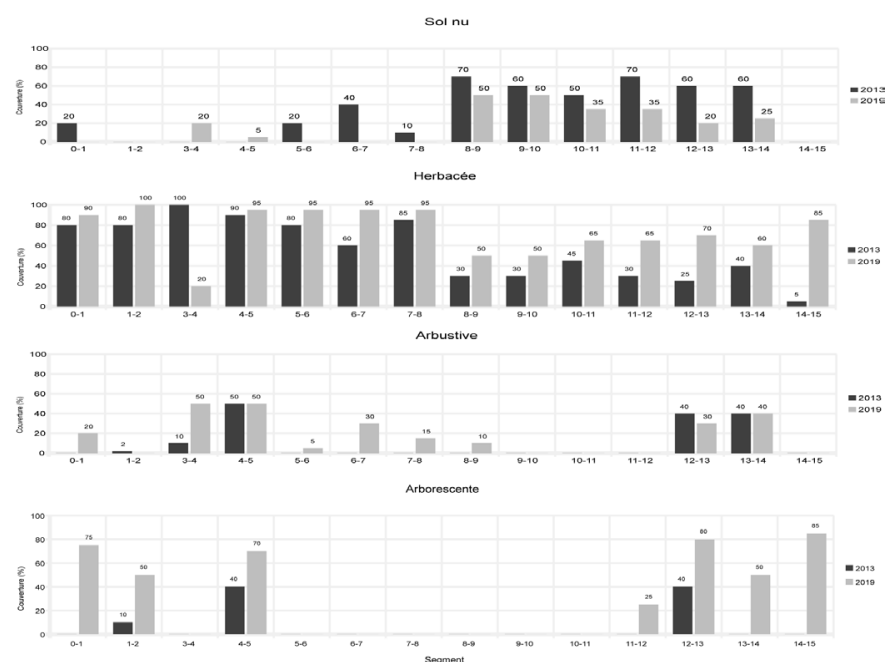

Figure 8 - Abondance de la couverture, en pourcentage, pour la strate arborescente, arbustive, herbacée et le sol nu, pour les années 2013 (noir) et 2019 (gris), dans un site alluvial (Les Boires, Pont-du-Château). Au-dessus de chaque colonne est affiché le pourcentage de la strate.
La strate arborescente est présente aux deux extrémités du transect (secteur de la boire puis forêt alluviale et secteur des rives de l'Allier). Au niveau de la végétation rivulaire (12-13, 13-14, 1415), on peut noter l'existence d'une strate arborescente qui n'était pas présente en 2013 (due à l'implantation d'une peupleraie pionnière) ; le segment 4-5 montre une augmentation de la couverture arborescente (traduisant la maturation d'une frênaieormaie déjà en place en 2013 ; habitat d'intérêt communautaire UE 91F0). Le développement de la strate arbustive en 2019 s'observe principalement au niveau des segments 6-7, 7-8, 8-9 (grèves colonisées par de jeunes pousses de Populus nigra L.). Elle traduit une dynamique vers la saulaie-peupleraie (éloignement de la nappe suite au déplacement de la rivière Allier). On constate aussi sur les grèves une augmentation nette de la couverture de la strate herbacée entre 2013 et 2019 (surtout segments 8-9 à 14-15) ; cela se fait aux dépend du sol nu.

On observe aussi que dans la boire asséchée (segment 0-1) la dynamique de la végétation est très rapide puisque qu'en l'espace de 7 ans se sont développés arbres et arbustes au détriment du sol nu.

Les informations sur les couvertures fournies par le logiciel permettent donc d'effectuer un suivi précis de la dynamique de la végétation.

\section{Foumiture d'un bilan taxonomique qualitatifentre différentes années}

Le logiciel fournit automatiquement un bilan taxonomique qualitatif entre plusieurs années, dans un même relevé ou dans chacun des segments d'une séquence. Cela permet de mieux comprendre les changements des valeurs des indices d'Ellenberg entre ces années.

Pour illustrer cette fonctionnalité on donne ici l'exemple du site de Monthiallier. II s'agit de landes subalpines de lisières (habitats d'intérêt communautaire UE 4030) et d'une hêtraie dite "subalpine » (habitat d'intérêt communautaire UE 9140). Le tableau 3 donne les espèces qui étaient présentes seulement en 2012 et celles seulement en 2019.

Tableau 3 - Liste des taxons « apparus » ou « disparus » dans la toposéquence mise en place à Monthiallier, entre 2012 et 2019.

\begin{tabular}{l|}
\hline \multicolumn{1}{c|}{2012} \\
\hline Betula pendula Roth \\
\hline Bistorta officinalis Delarbre \\
\hline Campanula rotundifolia $\mathrm{L}$. \\
\hline Dianthus seguieri Vill. subsp. pseudocollinus (P.Fourn.) Jauzein \\
\hline Dryopteris $x$ deweveri (J.T.Jansen) J.T.Jansen \& Wacht. \\
\hline Hieracium sp. L. \\
\hline Knautia basaltica Chass. \& Szabó var. foreziensis (Chass. \& Szabó) Breton-Sintès \\
\hline Lactuca plumieri (L.) Gren. \& Godr. \\
\hline Luzula multiflora (Thuill.) Arcang. \\
\hline Narcissus pseudonarcissus (L) Rouy \\
\hline Oxalis acetosella L. \\
\hline Ranunculus platanifolius L. \\
\hline Rubus idaeus $\mathrm{L}$. \\
\hline Sanguisorba officinalis L. \\
\hline Senecio cacaliaster Lam. \\
\hline Serratula tinctoria $\mathrm{L}$ subsp. monticola (Boreau) Berher \\
\hline Solidago virgaurea L. \\
\hline Thesium alpinum L. \\
\hline Veratrum album L. \\
\hline \\
\hline Carex umbrosa Host \\
\hline Hypochaeris maculata $\mathrm{L}$. \\
\hline Sorbus aria (L.) Crantz \\
\hline
\end{tabular}


On observe une « disparition " globale très importante de taxons en 2019 par rapport à 2012 (Tab. 3). En effet 18 taxons, présents en 2012, n'ont pas été revus en 2019. Inversement seulement 3 taxons, vus en 2019, ne l'avaient pas été en 2012. Parmi les taxons disparus, on compte de nombreuses espèces hygroclinophiles, comme Bistorta officinalis, Narcissus pseudonarcissus, Sanguisorba officinalis, Luzula multiflora et des taxons mésotrophiles de la classe phytosociologique des Mulgedio-Aconitetea Hadač \& Klika 1944, classe des hautes herbes subalpines, adeptes des sols profonds ou humides, relativement riches en nutriments, comme Lactuca plumieri, Ranunculus platanifolius, Senecio cacaliaster et Veratrum album. Une déduction possible de ces observations est qu'on assiste à une réduction naturelle de la congère (diminution de la durée et de l'épaisseur du manteau) provoquant la diminution ou la disparition de ces taxons. Le logiciel peut donc ainsi permettre de mettre en évidence des changements de végétation dus à des facteurs climatiques plus généraux.

\section{Variations par taxon}

La figure 9 montre par exemple l'évolution de l'abondance de la Ligulaire de Sibérie, dans la toposéquence permanente de suivi mise en place dans le marais de Fontfreyde, pour les années 2012 et 2019. Cette espèce est une relicte glaciaire en régression, elle est inscrite dans la Directive " Habitats-Faune-Flore" : annexes II et IV ; la Convention de Berne : annexe I ; c'est une espèce protégée au niveau national en France (annexe I) et est coté à I'UICN pour la France comme vulnérable.

\section{Ligularia sibirica - Herbacée}

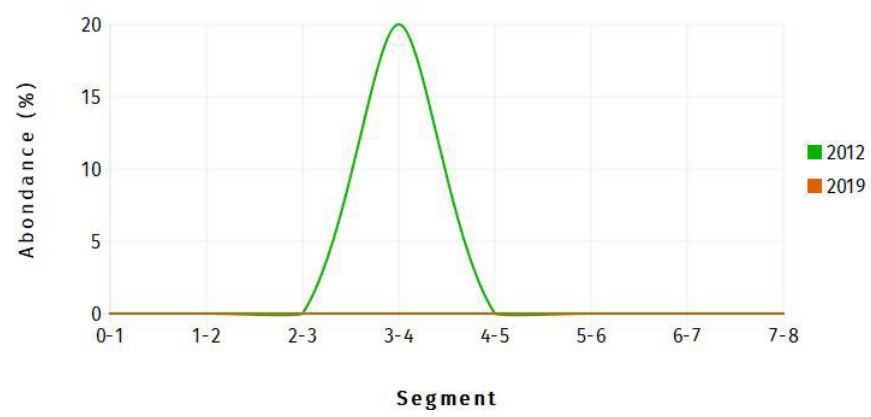

Figure 9 - Abondance, en pourcentage, de Ligularia sibirica (L.) Cass. Le long de la toposéquence. On constate que ce taxon n'était présent que dans le segment 3-4 en 2012 (20\%) et a complétement disparu de la séquence en 2019.

Les observations ont permis de constater sa disparition en 2019. La réduction de la période d'inondation du marais (paragraphe classe d'indice), a un impact négatif fort sur le développement et le maintien de la population. Ce facteur couplé à l'augmentation des valeurs de l'indice des températures (paragraphe valeurs d'indice), à la disparition d'autres espèces montagnardes (non présentée ici), et à une augmentation des valeurs de l'indice des nutriments (non présentée ici) le tout entraînant une compétition avec les hautes herbes de la mégaphorbiaie (Heinken-Šmídová \& Münzbergová 2012 ; Cîslariu et al. 2018), laisse à penser que la station de Ligulaire est en péril dans le marais de Fontfreyde.
A partir de cette observation, il a été décidé d'effectuer un comptage précis des pieds de Ligulaire dans l'ensemble du marais de Fontfreyde afin de pouvoir suivre, au plus juste, l'évolution de la population dans les années à venir. Un nouveau suivi de la toposéquence est prévu en 2024 afin de constater si les tendances de l'asséchement du marais et de l'augmentation des températures se confirment.

\section{Applications liées aux analyses agropastorales}

Un module agro-écologique complète le logiciel SurVegE. Pour chacun des segments du profil (ou des relevés isolés) il permet de calculer la valeur pastorale (VP') rapportée au \% de la strate herbacée, à partir des valeurs d'un indice spécifique de qualité fourragère (IS) attribuées à chaque taxon et de la fréquence spécifique de ceux-ci (FS). On peut ainsi en déduire le chargement en bétail adapté par hectare pour une période donnée (Loiseau 1989) et ainsi fournir cette référence de base pour la conduite du troupeau. L'indice spécifique de qualité fourragère (Daget \& Poissonet 1972, 2010) varie de 0 à 5 , avec 0 : espèce non fourragère (Thesium humifusum DC.) et 5 : très bonne fourragère (Dactylis glomerata L.). SurVegE fournit, dans ce module, les valeurs pastorales, un histogramme classant par affinité fourragère les taxons et il donne la possibilité de suivre la fréquence spécifique d'un taxon donné au cours du temps.

Une telle étude a été réalisée sur le puy de la Combegrasse. Ce puy était recouvert par une Pinède qui a été défrichée afin de remettre la mise en estive de la pelouse. Depuis 2014 un troupeau d'ovins pâture ces pelouses. La parcelle de suivi permanente a été mise en place en 2015 et le suivi a été effectué en 2020.

Le résultat principal est l'apparition d'espèces " bonnes fourragères " (IS=4 et 5, Dactylis glomerata L., Lotus corniculatus L., Poa pratensis L., etc.) et l'augmentation des valeurs de pourcentage des « moyennes fourragères » en 2020 par rapport à 2015 (IS= 1 à 3) (Fig. 10A). On remarque aussi que les pourcentages de terre nue/litière ont beaucoup diminué entre 2015 et 2020 . Confirmant ces tendances, la valeur pastorale augmente nettement en 2020 (Fig. 10B). Du point de vue de la valeur pastorale de ce parcours, on constate une amélioration de la qualité, on peut donc conclure à l'effet bénéfique général des pratiques pastorales actuelles.

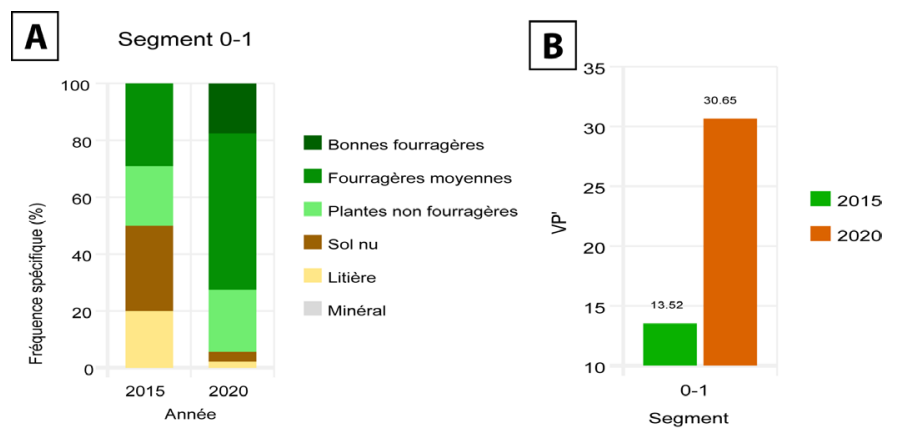

Figure 10 - A) Histogrammes comparatifs entre les années 2015 et 2020, des contributions spécifiques de chaque groupe d'espèces et terre nue/ litière ; B) histogramme représentant les valeurs pastorales déterminées pour chaque année. 
Cette étude sera poursuivie dans les années à venir, une attention particulière sera apportée aux signes d'un surpâturage avec apparition d'un phénomène d'érosion et donc d'augmentation des pourcentages de terre nue/litière ou au contraire d'un souspâturage avec apparition de ligneux colonisateurs.

\section{Conclusion}

SurVegE n'a pas pour objectif de définir de nouvelles méthodes d'analyse de la végétation, par contre il a l'avantage d'intégrer des outils largement utilisés dans une même application. II permet ainsi d'obtenir automatiquement, à partir des relevés effectués sur le terrain, les résultats relatifs au diagnostic et aux changements de végétation, sous forme de tableaux et illustrations. L'utilisateur peut ainsi exploiter facilement les résultats liés aux variables écologiques et manipuler un grand nombre de paramètres, tout en évitant des calculs fastidieux, permettant ainsi un gain de temps lors de l'exploitation des données.

L'utilisation des bio-indicateurs végétaux dans l'analyse comparative diachronique permet d'observer les changements intervenus au sein de la végétation. Avec cette approche même les faibles variations sont observables en l'espace de quelques années (Combegrasse 5 ans ; Les Boires 6 ans ; Fonfreyde, Monthiallier et puy Long 7 ans). Dans beaucoup d'exemples cités dans cet article, les segments des toposéquences permanentes étaient toujours rattachés au même habitat (ou à la même association végétale) lors du suivi, alors qu'on a pu constater des modifications de celui-ci. Cette approche permet donc de mettre en place rapidement une gestion fine et adaptée à chaque site.

Afin de pouvoir observer les variations de végétation, liées aux changements climatiques ou à la dynamique, il est nécessaire de suivre les parcelles pendant plusieurs dizaines d'années. II est donc primordial de les borner et géolocaliser rigoureusement car la comparaison diachronique ne fournira des résultats utilisables que si l'on suit exactement les mêmes segments au cours du temps. Si d'anciennes parcelles de suivis ont été bornées, géocalisées et que l'abondance des taxons présents a été relevée, il est tout à fait possible de rentrer les informations dans SurVegE afin de pouvoir continuer les suivis antérieurs. L'analyse des changements de végétation pourra alors s'effectuer à partir de la date de mise en place de ces parcelles permanentes et permettre ainsi de ne pas perdre les données collectées tout en poursuivant le suivi avec l'appui du logiciel.

On sait que, d'une manière générale, les résultats d'une comparaison diachronique entre les végétations observées sur une même parcelle peuvent dépendre beaucoup de l'observateur. On recommande donc une grande rigueur pour effectuer les relevés et attribuer la classe d'abondance la plus proche de la réalité, le guide d'utilisation du logiciel fournit d'ailleurs les précautions à prendre dans ce cas.

L'analyse des changements de végétation est un processus complexe et doit prendre en compte de nombreux facteurs ; en aucun cas SurVegE ne donne d'interprétation. II ne faut pas l'utiliser de manière simpliste en limitant la réflexion à l'observation d'un seul paramètre et pendant une période trop courte. Une telle approche pourrait s'avérer négative et par conséquent donner lieu à une gestion inappropriée.

SurVegE étant un logiciel évolutif, il pourra dans l'avenir être complété et amélioré par de nouveaux modules. Pour se procurer le logiciel il faut se rendre sur le site d'UniVegE (https://herbiers. uca.fr/version-francaise/survege) pour obtenir les modalités d'achat d'une clé de licence avec laquelle sera fourni le guide d'utilisation. Une formation sur la mise en place des parcelles permanentes sur le terrain et l'utilisation du logiciel en salle, sera également proposée.

\section{Remerciements}

SurVegE a été mis au point et testé sur plusieurs parcelles permanentes de différentes collectivités territoriales : le Conseil départemental du Puy-de-Dôme, Clermont Auvergne Métropole et le Parc des Volcans d'Auvergne, que nous tenons à remercier ici.

La mise au point du logiciel a bénéficié du soutien financier du ministère de la transition écologique et solidaire, de la fédération de recherche en environnement de l'université Clermont Auvergne et de l'initiative du gouvernement français IDEX-ISITE 16-IDEX-0001 (CAP 20-25) attribué au pôle universitaire de Clermont-Ferrand.

\section{Références}

Bardat J., Bioret F., Botineau M., Boullet V., Delpech R., Gehu J.-M., Haury J., Lacoste A., Rameau J.-C., Royer J.-M., Roux G. \& Touffet J., 2004. Prodrome des végétations de France. Coll. Patrimoines naturels, 61. Muséum national d'histoire naturelle, Paris, $171 \mathrm{p}$.

Bartoli M., Tran-Ha M., Largier G., Dumé, G. \& Larrieu L., 2000. Écoflore, un logiciel simple de diagnostic écologique. Revue forestière française, t. LII, $n^{\circ} 6: 530-547$.

Bensettiti F. \& Trouvilliez J., 2009. Rapport synthétique des résultats de la France sur l'état de conservation des habitats et des espèces conformément à l'article 17 de la directive habitats. Rapport SPN 2009/12, MNHN-DEGB-SPN, Paris. https://inpn.mnhn.fr/ telechargement/documentation/rapportage-directives-nature $16 / 11 / 2020$

Bensettiti F. \& Puissauve R., 2015. Résultats de l'état de conservation des habitats et des espèces dans le cadre de la directive Habitats-Faune-Flore en France. Rapportage "Article 17". Période 2007-2012. Service du patrimoine naturel, Muséum national d'histoire naturelle, Paris. https://inpn.mnhn.fr/ telechargement/documentation/rapportage-directives-nature $16 / 11 / 2020$

Braun-Blanquet J., 1932. Plant sociology, the study of plant communities. Transl. G.D. Fuller \& H.S. Conard. McGraw-Hill, New York : 476 p.

Braun-Blanquet J., 1964. Pflanzensoziologie Gründzuge der Vegetationskunde. Springer 3rd ed. Vienna, 865 p. https://doi. org/10.1007/978-3-7091-8110-2

Cîșlariu A., Mânzu C. \& Zamfirache M., 2018. Habitat requirements and germination performance of some relict populations of Ligularia sibirica (Asteraceae) from Romania. Plant Ecology and Evolution, 151(3) : 314-326. https://doi.org/10.5091/ plecevo.2018.1422.

Clair M., Gaudillat V., Michez N. \& Poncet R., 2019. HABREF v5.0, 
référentiel des typologies d'habitats et de végétation pour la France - Guide méthodologique. UMS PatriNat, AFB-CNRS-MNHNI, 95p.

Chytrý M., Hejcman M., Hennekens S. \& Schellberg J., 2009. Changes in vegetation types and Ellenberg indicator values after 65 years of fertilizer application in the Rengen Grassland Experiment, Germany. Applied Vegetation Science 12 : 167-176. https://doi-org.ezproxy.uca.fr/10.1111/j.1654109X.2009.01011.x

Daget P. \& Poissonet J., 1972. Un procédé d'estimation de la valeur pastorale des pâturages. Fourrages, 49 : 31-39.

Daget P. \& Poissonet J., 2010. Prairies et pâturages ; Méthodes d'étude de terrain et interprétations. CNRS/Cirad 955 p. https://umr-selmet.cirad.fr/publications-et-ressources/documents-techniques

Dierssen K., 2001. Distribution, ecological amplitude and phytosociological characterisation of European bryophytes. Bryophytorum Bibliotheca 56 : 1-289.

Duvigneaud P., 1946. La variabilité des associations végétales. Bulletin de la Société Royale de Botanique de Belgique, 78 : 107134.

Ellenberg H., Weber H. E., Düll R., Wirth V., Werner W., \& Paulißen D., 1992. Zeigerwerte von Pflanzen in Mitteleuropa. Scripta Geobotanica, 18 : 1-258.

Gargominy O., Tercerie S., Régnier C., Ramage T., Dupont P., Vandel E. et al., 2018. TAXREF v12.0, référentiel taxonomique pour la France. Muséum national d'Histoire naturelle, Paris. https:// inpn.mnhn.fr/telechargement/referentielEspece/taxref/12.0/ menu

Gaudin S., 2012. Apports des bases de données de valeurs indicatrices pour la validation et l'amélioration de groupes d'espèces indicatrices forestières. Bulletin de la Société d'Étude des Sciences Naturelles de Reims, 26 : 3-16.

Gégout J.-C., Coudun C., Bailly G. \& Jabiol B., 2005. EcoPlant: a forest site database linking floristic data with soil and climate variables. Journal of Vegetation Science, 16(2) : 257-260.

Hawkes J., Pyatt D. \& White I., 1997. Using Ellenberg indicator values to assess soil quality in British forests from ground vegetation: A Pilot Study. Journal of Applied Ecology, 34(2) : 375387. https://doi.org/10.2307/2404883

Heinken-Šmídová A. \& Münzbergová Z., 2012. Population dynamics of the endangered, long-lived perennial species, Ligularia sibirica. Folia Geobotanica, 47(2) : 193-214.

Hennekens S.M. \& Schaminée J.H.J., 2001. TURBOVEG, a comprehensive data base management system for vegetation data. Journal of Vegetation Science, 12 : 589-591.

Hill M.O. \& Carey P.D., 2009. Prediction of yield in the Rothamsted Park Grass Experiment by Ellenberg indicator values. Journal of Vegetation Science, 8(4) : 579-586. https://doi-org.ezproxy.uca. $\mathrm{fr} / 10.2307 / 3237210$

Julve Ph., 2017. Baseflor. http://philippe.julve.pagesperso-orange. $\mathrm{fr} /$ catminat.htm

Loiseau P., 1989. Signification et limite de l'indice de valeur pastorale pour le diagnostic de la valeur agricole des pâturages en moyenne montagne humide. Colloque Phytosociologique XVI, «Phytosociologie et pastoralisme», Paris 1988 : 411-428.

Mucina L., Bültmann H., Dierßen K., Theurillat J.-P., Raus T., Čarni A. et al., 2016. Vegetation of Europe: hierarchical floristic classification system of vascular plant, bryophyte, lichen, and algal communities. Applied Vegetation Science, 19 (Suppl. 1) :
3-264.

Société française de Phytosociologie. http://www.phytosocio. org/\#/pvf2 06/04/2021

Roux C., 2017. De la Limagne à la chaîne des Puys - Approche analytique intégrative pour l'étude des végétations actuelles et potentielles en moyenne montagne tempérée. Editions Revoir, thèse d'université Clermont Auvergne, 339 p. + Annexes. HAL Id : tel-02151595, version 1

Thébaud G., Roux C., Delcoigne A. \& Petel G., 2012. Contribution à une révision des bas marais acides d'Europe tempérée occidentale. Phytocoenologia, 42(1-2) : 67-98.

Wikum D.A. \& Shanholtzer G.F., 1978. Application of the BraunBlanuet cover-abundance scale for vegetation analysis in land development studies. Environmental Management 2 : 323-329. https://doi.org/10.1007/BF01866672 